Tuider, Elisabeth; Huxel, Katrin

\title{
Männlichkeit und die Übernahme von care-work im Migrationskontext
}

Moser, Vera [Hrsg.]; Pinhard, Inga [Hrsg.]: Care - wer sorgt für wen? Opladen u.a. : Verlag Barbara Budrich 2010, S. 87-98. - (Jahrbuch Frauen- und Geschlechterforschung in der Erziehungswissenschaft; 6)

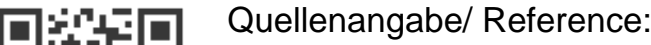

Tuider, Elisabeth; Huxel, Katrin: Männlichkeit und die Übernahme von care-work im

Migrationskontext - In: Moser, Vera [Hrsg.]; Pinhard, Inga [Hrsg.]: Care - wer sorgt für wen? Opladen u.a. : Verlag Barbara Budrich 2010, S. 87-98 - URN: urn:nbn:de:0111-opus-82421 - DOI:

10.25656/01:8242

https://nbn-resolving.org/urn:nbn:de:0111-opus-82421

https://doi.org/10.25656/01:8242

in Kooperation mit / in cooperation with:

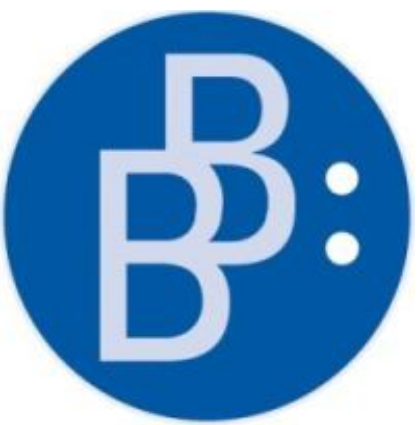

https://www.budrich.de

\section{Nutzungsbedingungen}

Gewährt wird ein nicht exklusives, nicht übertragbares, persönliches und beschränktes Recht auf Nutzung dieses Dokuments. Dieses Dokument is ausschließlich für den persönlichen, nicht-kommerziellen Gebrauch bestimmt. Die Nutzung stellt keine Übertragung des Eigentumsrechts an diesem Dokument dar und gilt vorbehaltlich der folgenden Einschränkungen Auf sämtlichen Kopien dieses Dokuments müssen alle Urheberrechtshinweise und sonstigen Hinweise auf gesetzlichen Schutz beibehalten werden. Sie dürfen dieses Dokument nicht in irgendeiner Weise abändern, noch dürfen Sie dieses Dokument für öffentliche oder kommerzielle Zwecke vervielfältigen, öffentlich ausstellen, aufführen, vertreiben oder anderweitig nutzen.

Mit der Verwendung dieses Dokuments erkennen Sie die Nutzungsbedingungen an.

\section{Terms of use}

We grant a non-exclusive, non-transferable, individual and limited right to using this document.

This document is solely intended for your personal, non-commercial use. Use of this document does not include any transfer of property rights and it is conditional to the following limitations: All of the copies of this documents must retain all copyright information and other information regarding legal protection. You are not allowed to alter this document in any way, to copy it for public or commercial purposes, to exhibit the document in public, to perform, distribute or otherwise use the document in public.

By using this particular document, you accept the above-stated conditions of use.

\section{Kontakt / Contact:}

peDOCS

DIPF | Leibniz-Institut für Bildungsforschung und Bildungsinformation Informationszentrum (IZ) Bildung

E-Mail: pedocs@dipf.de

Internet: www.pedocs.de

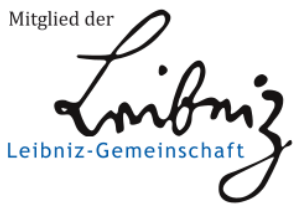


Care - Wer sorgt für wen? 
Jahrbuch der

Frauen- und Geschlechterforschung

in der Erziehungswissenschaft

herausgegeben von

Sabine Andresen

Rita Casale

Edgar Forster

Edith Glaser

Vera Moser

Annedore Prengel

Barbara Rendtorff

Beirat

Birgit Althans, Berlin

Eva Borst, Mainz

Eva Breitenbach, Osnabrück

Bettina Dausien, Bielefeld/München

Isabell Diehm, Bielefeld

Hannelore Faulstich-Wieland, Hamburg

Carola Iller, Heidelberg

Marita Kampshoff, Schwäbisch Gmünd

Margret Kraul, Göttingen

Andrea Liesner, Hamburg

Susanne Maurer, Marburg

Astrid Messerschmidt, Darmstadt

Inga Pinhard, Frankfurt

Folge 6/2010 
Vera Moser

Inga Pinhard (Hrsg.)

\section{Care - Wer sorgt für wen?}

Verlag Barbara Budrich

Opladen \& Farmington Hills, MI 2010 
Bibliografische Information der Deutschen Nationalbibliothek

Die Deutsche Nationalbibliothek verzeichnet diese Publikation in der Deutschen

Nationalbibliografie; detaillierte bibliografische Daten sind im Internet über

http://dnb.d-nb.de abrufbar.

Gedruckt auf säurefreiem und alterungsbeständigem Papier.

Alle Rechte vorbehalten.

(C) 2010 Verlag Barbara Budrich, Opladen \& Farmington Hills, MI

www.budrich-verlag.de

\section{ISBN 978-3-86649-323-0}

Das Werk einschließlich aller seiner Teile ist urheberrechtlich geschützt. Jede Verwertung außerhalb der engen Grenzen des Urheberrechtsgesetzes ist ohne Zustimmung des Verlages unzulässig und strafbar. Das gilt insbesondere für Vervielfältigungen, Übersetzungen, Mikroverfilmungen und die Einspeicherung und Verarbeitung in elektronischen Systemen.

Umschlaggestaltung: disegno visuelle kommunikation, Wuppertal - www.disenjo.de Druck: paper \& tinta, Warschau

Printed in Europe 


\section{Inhalt}

\section{Editorial}

Vera Moser/Inga Pinhard

Care: Wer sorgt für wen?

\section{Gastbeitrag}

Nel Noddings

Care Ethics, Caregiving, and Global Caring.

\section{Beiträge}

Micha Brumlik

Ethische Gefühle: Liebe, Sorge und Achtung

Marianne Friese

Die ,Arbeit am Menschen`. Bedarfe und Ansätze der

Professionalisierung von Care Work

Sabine Toppe

Care-Ethik und Bildung - Eine neue ,Ordnung der Sorge` im Rahmen von Ganztagsbildung?.

Elisabeth Tuider/Katrin Huxel

Männlichkeit und die Übernahme von care work im Migrationskontext.

Anne-Christine Kunstmann

Familiale Pflege als Angelegenheit der Frauen? Diskursive Deutungen zur Zukunft der Altenfürsorge und -pflege. 


\section{Aus der Forschung}

Celine Camus

Is von der Leyen really going too far?

Hildegard Macha/Susanne Gruber

Spielplatz der Exzellenz: Die Kultur der Sorge an Hochschulen.....

Anke Spies

In Sorge um die ,Kinder von morgen“? - ein pädagogisch motiviertes Instrument und seine Konsequenzen.

Susanne Gruber/Dieter Jaufmann

Kindertagesbetreuung: Eine strategische Variable bei innerfamiliären und gesamtgesellschaftlichen Veränderungsprozessen.

\section{Tagungsberichte}

Heike Kahlert

Doing family - doing difference (Hannover 2007)

Erna Appelt/Maria A. Wolf

Who Cares? Betreuung und Pflege in Österreich - eine geschlechterkritische Perspektive (Innsbruck 2009).

Thomas Viola Rieske/Irina Schmitt

Gender: Regulation and Resistance in Education (London 2009)

Laura Nina Paul

Care und Migration (Frankfurt 2009)

\section{Rezensionen}

Vera Müncher

Rezension zu: Martina Heitkötter/Karin Jurczyk/Andreas Lange/Ute Meyer-Gräwe (Hrsg.): Zeit für Beziehungen?

Veronika Magyar-Haas

Rezension zu: Bettina Hünersdorf: Der klinische Blick in der sozialen Arbeit. Sytemtheoretische Annäherungen an eine Reflexionstheorie des Hilfesystems 
Sebastian Winter

Rezension zu: Claudia Bruns: Politik des Eros.

Mechthild Bereswill

Rezension zu: Hans Günther Homfeldt/Wolfgang Schröer/Cornelia

Schweppe (Hrsg.): Soziale Arbeit und Transnationalität.

AutorInnenhinweise

Zum Jahrbuch Frauen und Geschlechterforschung in der Erziehungswissenschaft.

Ankündigung der nächsten Bände

Band 7

Band 8 


\title{
Männlichkeit und die Übernahme von care-work im Migrationskontext
}

\author{
Elisabeth Tuider/Katrin Huxel
}

\begin{abstract}
s
Das in Deutschland vorherrschenden Bild von „dem Migranten“ ist einerseits durch Gewalt und patriarchale Einstellungen sowie andererseits durch Ehre und einer Verantwortung gegenüber der Großfamilie bestimmt. Diesen dominanten Bildern über Männer mit Migrationsbiographie entgegen zeigen wir in unserem Beitrag anhand eines biographischen Interviews wie der Migrationskontext als Aushandlungs- und Möglichkeitsraum fungiert. In diesem kann sich Männlichkeit zum einen in Auseinandersetzung mit den vorherrschenden Normen und Zuschreibungen und zum anderen durch die Übernahme von care-work neu konstituieren.
\end{abstract}

Masculinity and the assumption of the responsibility of care work in the context of migration

The prevailing image of "the male migrant" in Germany is shaped by the notion of violence and patriarchal attitudes on one hand, as well as honour and responsibility concerning the extended family on the other. This article argues against these dominant concepts of men with biographies of migration and shows, based on biographical interviews, how the context of migration is constructed as space of negotiations and possibilities. Within this, masculinity can be newly constituted in two ways: through rethinking prevailing norms and attributions and assuming the responsibility of care work.

\section{Das Bild von ,dem Migranten“"}

\begin{abstract}
„Warum sollte er [Maqsod] auf die einzige Macht, die er besitzt, auf die Macht des Mannes, mit der er als Sohn über Schwester und Mutter verfügt, über jede Frau und auch seine zukünftige - warum sollte er ausgerechnet auf diesen Respekt verzichten? (...) Jungs wie Maqsod wissen, dass einzig die Tradition ihrer Heimat sie in Deutschland zu umhegten Herrschern macht." (Reich in: Stern 33/2006: 37)

Was in dieser Darstellung in einem Stern-Artikel von 2006 beispielhaft deutlich wird, ist das vorherrschende Bild von „dem Migranten“, das in weiten Teilen der (medialen) deutschen Öffentlichkeit und auch der wissenschaftlichen Forschung von Vorurteilen durchzogen ist. Während in den 1960er und
\end{abstract}


1970er Jahre die Figur des „Gastarbeiters“ prägend war, also desjenigen Ausländers, der heute kommt und morgen wieder gehen soll, können die 1980er Jahre mit „Kulturdifferenz“ und dem damit diskursiv immer verbundenen „Kulturkonflikt“ gelabbelt werden. Erst Ende der der 1990er Jahre erfolgte ein Perspektivenwechsel, denn Deutschland hatte sich endlich als „Einwanderungsland“ anerkannt und damit eröffnete sich ein neuer Fokus hin zur Thematisierung von Rassismus, Macht und Gewalt in Ausländerrechts- und Flüchtlingsfragen.

Von Frauen, von Arbeitsmigrantinnen, war in den 60ern kaum die Rede, und wenn von ihnen die Rede war, dann als „Weib und Mutter“ oder als ,in der Fremde Zurückgebliebene“. Mit den 80er Jahren veränderte sich zwar die Wahrnehmung von Frauen mit Migrationsbiographie, diese folgte aber - und tut dies in weiten Teilen bis heute - dem Bild der in jeder Hinsicht abhängigen Ehefrau. Denn trotz gegenläufiger empirischer Befunde werden ausländische Frauen immer wieder zu Hausfrauen und Müttern stilisiert, obwohl ihre Erwerbsbeteiligung bereits Anfang der 1970er mit rund 70\% weitaus höher als diejenige der deutschen Frauen (mit 47\%) lag.

Ende der 1990er Jahre trat die Radikalisierung v.a. von Männern mit Migrationsbiographie in den Mittelpunkt des öffentlichen Interesses. Das Projekt des „Multikulturalismus“ - in Deutschland ohnehin nur von einer kleinen Gruppe Engagierter ernsthaft betrieben und nie politisches Programm - wurde für gescheitert erklärt und mediale Diskursivierungen folgten nun der Gleichsetzung: ,Migrationshintergrund=gewaltbereit=kriminell=religiös=fundamentalistisch. Das Geschlechterverhältnis und damit die Konstruktion von Männlichkeit und Weiblichkeit erhält nun in den ethnisierenden Diskursen als Ort der Zuschreibung herausragende Bedeutung (vgl. dazu Gemende u.a. 2007; Tunc 2009). Dominante Themen dieses ethnisierenden Geschlechterdiskurses sind: „Ehrenmord“, „Zwangsheirat“ und „Gewalt gegen Frauen“. Dabei wird davon ausgegangen, dass „Mädchen bzw. Frauen hilflose Opfer einer misogynen Unterdrückung seien und einer totalen Kontrolle durch despotische Väter, Brüder, Ehemänner oder andere, männliche Verwandte unterlägen. [...] Bezogen auf Jungen bzw. Männer ist die Wahrnehmung [...] vom Klischeebild des 'Macho' oder 'Pascha' vorstrukturiert.“(Weber 2005: 154)

In dem „Bild von dem Migranten“ wird der Männlichkeitsentwurf von ethnisierten Männern als durch Gewalt, Drogen, Frauenverachtung und protziges Auftreten durchdrungen skizziert. Zugleich sei die Maskulinitätsnorm v.a. junger Türken - bestimmt von sog. traditionellen Werten wie Ehre, einer Beschützerfunktion und Versorgungsverantwortung gegenüber der Großfamilie. An der kaum bzw. erst in den letzten Jahren bearbeiteten Schnittstelle von Migrations- und Väterforschung führt Michael Tunc das Stereotyp der öffentlichen Negativdiskurse in Hinblick auf den türkischstämmigen Mann und Vater aus: „Speziell der Vater aus der Türkei gilt als strenger, patriarchal- 
autoritärer Typ, der nicht integrierbar und im Wesentlichen darauf bedacht ist, die Ehre seiner Familie auch unter Anwendung von körperlicher Gewalt zu schützen, die weiblichen Mitglieder der Familie unterdrückt und die Integration der Ehefrauen und Töchter verhindert." (Tunc 2007: 34) In der Wahrnehmung von „fremden Männlichkeiten“ dominiert dabei die Wahrnehmung des ethnisch, kulturell, national 'Anderen'. Entgegen diesen Bildern über „den Migranten“ möchten wir in unserem Beitrag der Frage nachgehen, wie sich Männlichkeit im Migrationskontext durch die Übernahme von careAufgaben (neu) konstituiert. Den Migrationskontext möchten wir dazu als Verhandlungs- und Aushandlungsraum definieren, in dem sowohl Geschlecht und das Geschlechterverhältnis als auch die ethnische Zugehörigkeit in Frage gestellt werden. Speziell in den Arbeits- und Familienverhältnissen, so unsere weitere These, können - und müssen zum Teil - Anpassungen, Verschiebungen und Umdefinitionen von Bindungen und Zugehörigkeiten erfolgen. Denn die Effekte der Migration und das Leben in der Einwanderungsgesellschaft Deutschland wirken sich auf die Konstitution von Männlichkeit aus:

„Der eingewanderte Mann bleibt nicht der Mann, der er vor der Einwanderung war. (...) Seine vormals hegemoniale Männlichkeit kann sich in eine marginalisierte verkehren, die noch hegemoniale Tendenzen aufweisen kann, aber gesellschaftlich keine Autorität mehr besitzt und der normativen Kontrolle, vorwiegend des eigenen Geschlechts unterliegt." (Herwartz-Emden 2000: 38)

Diese Thesen werden wir am Beispiel von Auszügen aus einem biographischen Interview veranschaulichen.

\section{Migration und Care}

Auf der sozialstrukturellen Ebene erfahren nach Deutschland Migrierte eine Dequalifizierung und Aberkennung im Herkunftsland erworbener Bildungszertifikate, sie erwerben in Deutschland signifikant niedrigere Bildungsabschlüsse und sie brechen häufiger die (Haupt)Schule ab (King/Koller 2006; Diefenbach 2007). Im Ergebnis sind Migranten häufiger als ihre deutschen männlichen Kollegen von Arbeitslosigkeit und von Ausgrenzung auf dem Arbeitsmarkt betroffen und sie arbeiten häufiger in den unteren Segmenten des Arbeitsmarktes, der von schlechter Bezahlung und hoher Prekarität gekennzeichnet ist, d.h. sie arbeiten in der Leiharbeit und den Minijobs, den 400€Jobs und der Zeitarbeit; Darüber hinaus ist Männern mit Migrationsbiographie - entsprechend geschlechtsspezifischer Zuschreibungen - der ethnisch segmentierte Arbeitsbereich der schlecht bezahlten und oftmals illegalisierten care-Arbeit weitgehend verschlossen. 
In der Migrations- und auch in der Transmigrationsforschung werden die „globalen Betreuungsketten“ (Hochschild 2000) sowohl als push- als auch als pull-Faktor der Migration verhandelt. Denn es ist einerseits die Verantwortlichkeit von Müttern für das soziale und emotionale Wohl und das Überleben der Kinder, also die Ausübung von care, das als einer der spezifischen Wanderungsgründe für Frauen behandelt wird. Andererseits ist es, wie bspw. Arlie Hochschild oder Saskia Sassen zeigen, gerade die weibliche Arbeitskraft, die in der globalen Ökonomie gefragt ist. Denn Migrantinnen sind in der neuen globalen Dienstleistungsindustrie v.a. als „,neue Dienstmädchen“ (Lutz 2007) notwendig, da die steigende Bildungs- und Erwerbsbeteiligung der Frauen in den Industrieländern zu einem wachsenden Bedarf an Arbeitskräften im Bereich der privaten Haushalte geführt hat. Es ist, so Lutz, la putzfrau, die die ehemalige von Gisela Bock und Barbara Duden entzauberte „Arbeit aus Liebe" (Bock/Duden 1977) als care-takerin in westlichen und deutschen Haushalten übernimmt. Dabei wird die reproduktive Arbeit von einer weiblichen Hand an eine andere übergeben - care bleibt aber weiblich konnotiert.

Carlota Solé u.a. (2007) resümieren zur Übernahme von care-Aufgaben im Migrationskontext zwei Szenarien: Die Migration des Mannes, des Vaters, entspricht der Geschlechterzuschreibung als Familienernährer und -versorger. Wenn allerdings die Frau (und Mutter) den Migrationsprozess initiiert und dadurch zur Familienernährerin wird, kann dies auf ihrer Seite einen Prozess des Empowerments auslösen. Es kann über das Empowerment hinaus auch die traditionelle Rolle des Mannes völlig in Frage gestellt werden, oder wie Sonia Parella es formuliert: „The hegemonic patriarchal model is thrown into crisis.“ (2008: 10). Die Migration stellt in jedem Fall einen Wendepunkt für die Familien- und Geschlechterkonstellationen dar.

Die eingangs skizzierten diskursiven Zuschreibungen an ethnisierte Männlichkeiten implizieren eine patriarchale und despotische Männlichkeitsund Vaterschaftskonzeption; Die dargestellten sozialstrukturellen Benachteiligungen von Migranten weisen wiederum darauf hin, dass Männer mit Migrationsbiographie hegemoniale Konstruktionsmodi von Männlichkeit (vgl. Scholz 2004), nämlich Brotverdiener und Familienernährer zu sein, nicht anwenden können. Männer mit Migrationsbiographie verfügen über geringeres ökonomisches, kulturelles (Bildungskapital) und weniger soziales Kapital. Anja Weiß (2001) begründet die niedrigere Position von Migrierenden im sozialen Raum auch darüber, dass das Kapital ethnisierter Minderheitenangehöriger, bspw. in Form von Sprachkompetenzen, delegitimiert und entwertet wird. Wir möchten nun im nächsten Teil unseres Beitrages die Frage aufgreifen, wie sich die Übernahme von care-work im Migrationskontext verändert und welche Auswirkungen dies auf Männlichkeit hat. Anhand eines biographischen Interviews wollen wir zeigen, dass Männlichkeit im Migrationskontext sich sowohl in der Auseinandersetzung mit den Normen und Zuschreibungen der Ankunftsgesellschaft (=Deutschland) als auch in Ausein- 
andersetzung mit den Normen und Erwartungen der Herkunftsgesellschaft herstellt. Männlichkeit in der Migrationssituation zeichnet eine mehrfache Zugehörigkeit und damit zusammenhängend eine Auseinandersetzung mit anderen Männern und Vätern aus, wie es auch Michael Tunc am Beispiel einer biographischen Erzählung nachzeichnen konnte: „Ich weiß nicht, also, ob viele türkische Väter [2] gleich Ehemänner, ob die halt, vor ihren Frauen flüchten zu Hause, oder, nur also viel, so mit den Kindern irgendwas machen, also ich hab noch nie [lacht] nen türkischen Vater gesehen also, ist selten, noch nie wär zu viel gesagt, aber, das die da, sich aufs Fahrrad setzen und mit ihren Kindern, fahren, hab ich nicht." (Hakan in Tunc 2009: 122)

Die Biographieforschung fokussiert individuelle Lebensgeschichten vor dem Hintergrund, dass in der „Konkretheit des individuellen Falls Allgemeingültiges [...] verborgen" ist (Alheit 1992: 20). Sie zielt darauf, die Spuren des gesellschaftlichen Allgemeinen in den einzelnen Biographien zu rekonstruieren, denn die methodologische Basisannahme der Biographieforschung ist , dass den Erzählungen generative Strukturen „in einer gemeinsam geteilten Wirklichkeit" (Rosenthal/Fischer-Rosenthal 2003: 457) zugrunde liegen, die in der Interaktion zwischen dem Forschenden und dem Erzählenden belebt, aufgedeckt und wissenschaftlich rekonstruiert werden können. Dabei wird eine Dialektik von Individuellem und Gesellschaftlichem vorausgesetzt, die in der biographischen Analyse in den Blick genommen wird. Denn Biographieforschung analysiert das Zusammenspiel von sozialer Struktur und kollektivem Regelsystem einerseits und individueller Sinnkonstruktion im spezifischen (Forschungs-) Setting andererseits. Es geht demnach um die Frage, wie Individuen gesellschaftliche und milieuspezifische Bedingungen zu einem je spezifischen historischen Zeitpunkt sich aneignen und verarbeiten und um deren Reaktualisierung im Forschungssetting (vgl. FischerRosenthal/Rosenthal 1997: 405).

\section{Biographie im Migrationskontext: das Beispiel Mustafa}

Mustafa wird 1944 in einer der Provinzhauptstädte Süd-Ost-Anatoliens geboren, er absolviert eine Lehramts-Ausbildung und heiratet 1968. Zum Zeitpunkt des Interviews ist er 59 und seine Frau 54 Jahre alt.

Nachdem das jüngere der beiden Kinder an Masern starb, entscheiden sich er und seine Frau zur Migration nach Deutschland und sie stellen Anträge dafür. Mustafas Frau erhält als erste ihre Ausreisebewilligung und geht nach Süddeutschland. Mustafa bleibt zusammen mit der ältesten Tochter in der Türkei.

Der im Oktober 1973 verhängte Anwerbestopp durchkreuzt die Pläne der 
Familie, denn es wird plötzlich unklar, ob und wie Mustafa zu seiner Frau nach Deutschland kommen kann. Bereits 1974 verfügt er über ein gültiges Studentenvisum für die Einreise, das ihm aber nicht erlaubt einer bezahlten Tätigkeit nachzugehen. Nach einigen Jahren in Deutschland erhält Mustafa die Möglichkeit, als Lehrer - zunächst im muttersprachlichen Ergänzungsunterricht - zu arbeiten. Auch seine Frau erhält schließlich die Lehrbefugnis. Heute sind beide an deutschen Schulen als Lehrkräfte tätig. Sie haben zwei erwachsene Töchter, die älteste Tochter ist mit einem deutschen Mann verheiratet und arbeitet als Ärztin, die jüngere Tochter studiert.

Mustafa muss - verursacht durch die besonderen Umstände der Migrationsituation - im Interview seine Präsentation von Männlichkeit immer wieder umdeuten und anpassen. In der Türkei ist er nach der Migration seiner Frau eine Zeitlang quasi alleinerziehender Vater, in Deutschland kann er die Aufgabe des Familien(mit)ernährers zeitweise nicht oder nur unzureichend ausfüllen. Die migrationsbedingten äußeren Umstände schränken also seine Möglichkeiten, sich an hegemonialen Konstruktionsmodi zu orientieren, ein. Mustafa erlebt gerade die Einschränkungen der Anfangszeit in Deutschland als entwertend und verbalisiert diese Erfahrung folgendermaßen:

„Für eine 30 jährige Mann, der Lehrerausbildung in der Türkei abgeschlossen hat, nach Deutschland kam, du bist erst einmal als Nichts-Wissender, Nicht-Nützlicher eh, eh abgestempelt, ne? Und obwohl dass du weißt, du kannst aber das nicht erzählen, was du kannst, da weißt du gar nichts. Du bist dumm, du bist wirklich stumm, du kannst nichts.“

Schon seine ersten Erfahrungen in Deutschland bettet er also in den Kontext von Männlichkeit ein, indem er sich eindeutig als „Mann“ positioniert. Aber als ,30 jähriger Mann“ wird er durch die Migration entmachtet, deklassiert und ,abgestempelt“. Wie im Folgenden gezeigt wird, referiert Mustafa in seiner Erzählung immer wieder auf seine männliche Position, dabei wird die Vielschichtigkeit seiner Vorstellungen, aber vor allem seines (Er)Lebens von Männlichkeit im Kontext seiner biographischen Erfahrungen deutlich.

\section{Männlichkeit in der biographischen Erzählung}

Wie eben gezeigt, thematisiert Mustafa seine Männlichkeit einerseits als entwertet und von hegemonialen Konstruktionsmodi ausgeschlossen. Seine biographische Bearbeitung der Phase, in der seine Frau die Familienernährerin war, zeigt, dass er sich einerseits , auf ein Nichts' reduziert erlebte. Andererseits gelingt ihm jedoch eine erfolgreiche Anpassung an diese strukturellen und biographischen Bedingungen und eine Transformation seines ,doing masculinity' und der familiären Arbeitsteilung. Doch nicht erst aufgrund der 
strukturellen Anforderungen wird Mustafa zum ,modernen', Geschlechter gerecht denkenden Mann (vgl. Zulehner/Volz 2009). Er präsentiert sich über die Reaktion auf diese veränderten Anforderungen hinaus in einer ,,partnerschaftlichen Männlichkeit“ und führt seine geschlechtergerechte Einstellung auf seine eigene Erziehung zurück. Damit verortet er sie in der Türkei, revidiert das vorherrschende Türkei-Bild in Deutschland und bezieht insbesondere indirekt auch Position gegen in Deutschland dominante Vorstellungen des türkischen Familienpatriarchen:

„Als wir uns kennen gelernt haben und auch heirateten und ehm, das eh was ich von Mama gelernt habe, hat das in der Ehe auch das praktiziert. Das, für mich ist es ehm keine Problem, bei Haushalt zu helfen zum Beispiel (ja). Ehm für mich ist es absolut, ich hab damit überhaupt kein Problem, wenn, eh, bedingt ihre Beruf und meine Frau woanders hingehen muss, und eh, der andere zusammenkommen und nachfolgen muss und eh, Seminare machen muss, und entweder hast du für deine Partner vertrauen oder hast du das nicht, ob sie berufstätig ist oder kein berufstätige Partner ist."

Mustafas Auseinandersetzung mit traditionellen Rollenvorstellungen und seine Umdeutung dieser sprechen für einen Wandel von Geschlechterverhältnissen, wie sie Volz und Zulehner $(1998,2009)$ festgestellt haben: es erfolgt eine (langsame) Abkehr vom Alleinernährermodell und eine Hinwendung zur Übernahme von Haus-, Familien- und Erziehungsarbeit (=care).

Obwohl Mustafa im Rahmen seiner Selbstbeschreibung dominante Diskurse über ,den türkischen Mann/Vater' nicht bestätigt, greift er an anderer Stelle affirmativ auf diese diskursiven Figuren zurück. Er entwirft ein Bild von ,typischen türkischen Familien', demonstriert in langen argumentativen Passagen sein Expertenwissen über, Türken in Deutschland' und betont, worin er und seine Familie sich von diesen unterscheiden. Im folgenden Interviewauszug beschreibt Mustafa die Reaktionen anderer aus der Türkei Migrierter auf seinen Erziehungsstil, deren autoritären und an Verboten orientierten Erziehungsstil und stellt dem seine eigene, im Gegensatz dazu liberale und offene Einstellung gegenüber:

“'Euer Kind, habe ich gehört geht mit Jungs in die Stadt.' (mhm) Davor, vor diese Rufmord einfach haben die Angst. Und eh, die haben so große Angst, und dann verlieren die halt den Maßstab bei dem Verbot. Und eh, dann sagen die verbieten dir alles. Und eh, ob die damit wirklich alles erreichen oder erreichen können, das eh, das bezweifle ich sehr stark. Wir haben das eh, ich kann sagen, wir haben das nicht so gemacht. Eh, das eh, jetzt kommt dann auch. Ich kann so eine Sache erzählen, die G. hat jemand kennengelernt, der weder Türke noch Muslime ist, und eh den ja, er ist ein Deutscher und ist auch eine Christ und eh, ja jetzt eh, aber habe ich am Anfang unseres Gesprächs auch erzählt, und für mich solche Sachen keine Rolle spielen, das die Entscheidung unserer Tochter ist (ja).“

Mustafa präsentiert den Umgang in der eigenen Familie, z.B. die Arbeitsteilung zwischen ihm und seiner Frau oder die Kindererziehung, als different von anderen türkischstämmigen Familien. Über diese Abgrenzung von 
, anderen Türken' und der Darstellung der eigenen Lebensweise als ,abweichend' davon kann Mustafa sich - den herrschenden Diskursen über türkische Männer/Väter zum Trotz - als ,moderner', an Care-work partizipierender Mann und aktiver Vater, aber auch als weltlich orientierter Türke und Muslim darstellen.

In Mustafas gesamter biographischer Selbstbeschreibung spielt Vaterschaft eine große Rolle. Schon früh führt er seine Töchter in die Erzählung ein, thematisiert die eigenen Erziehungsvorstellungen, aber auch die Entwicklungen und Erlebnisse seiner Kinder. Er wählt damit zentrale Themen, die auf der inhaltlichen Ebene nicht unbedingt für die narrative Konstruktion von Männlichkeit typisch sind. So stellt Scholz (2004) fest, dass Männlichkeit im Interview auf der inhaltlichen Ebene eher durch die De-Thematisierung familiärer Bereiche hergestellt wird. In Mustafas Fall findet die narrative Konstruktion von Männlichkeit jedoch auch und gerade in der Präsentation als Vater statt. Er betont dabei seinen hohen Bildungsanspruch, den er an seine Töchter hat und der sich beispielhaft in folgender Geschichte ausdrückt:

„Als die R. diese von zehn in die elf kam auf dem Gymnasium, ich hab der R. gesagt: ,R., du musst hier kein Abitur machen, wenn du keine Lust hast, machste keins. Und aber wenn du jetzt beginnst in der elf, ich verlange von dir vernünftige Abitur. Weil du die Kapazitäten hast. Du bist nicht dumm und ne so nicht irgendwie so larifari in die Schule gehen, nach drei Jahren ,Komm, Papa, ich hab nicht geschafft!'. Aber du kannst in diese drei Jahren auch eine Ausbildung machen'.“

In dieser Erzählung zeigt sich Mustafas aktive Teilnahme an der Erziehung seiner Töchter. Er ist nicht einfach ,nur' Familienernährer, sondern aktiv an zentralen Fragen der Erziehung beteiligt. Er spricht mit seiner Tochter, verhandelt mit ihr, stellt aber auch Ansprüche. Wie oben inszeniert er sich als partnerschaftliche Autorität und zeigt, dass seiner Erziehung demokratische Prinzipien zugrunde liegen und seine Töchter selbst Entscheidungen über ihr Leben treffen. Mustafa betont in dieser Sequenz einerseits sein Interesse an der Bildung seiner Tochter, andererseits gesteht er ihr aber auch zu, sich anders zu entscheiden. Mit der Präsentation als aktiver Vater mit demokratischen Erziehungsprinzipien und auch durch die Betonung seines Bildungsinteresses nimmt Mustafa erneut eine Abgrenzung zu anderen türkischstämmigen Männern mit Migrationsbiographie vor, die er aus einer Expertenposition heraus nicht nur als streng (s.o.), sondern auch als wenig an Bildung interessiert und wenig ehrgeizig beschreibt. Wieder referiert er damit auf Figuren dominanter Diskurse der Mehrheitsgesellschaft, von denen er sich und seine Familie abgrenzt.

Doch Mustafa grenzt die eigene Familie nicht nur von anderen ,ausländischen Familien“ ab, sondern unter Umständen auch von deutschen, wie er in folgender Erzählung andeutet:

„Na ja, eine andere Sache, als wir an der S.-Straße wohnten, das eine Sechsfamilienhaus da 
und da gegenüber wohnte eine andere Familie und eine deutsche Familie, wir waren nur eine einzige ausländische Familie von diese sechs. Und eh, unsere Kinder mussten ihre Hausaufgaben machen, während die anderen Kinder draußen spielten. Sagte unsere Nachbarin zu mir direkt, sagte: 'Ja, Mustafa du setzt deine Kinder unter Druck, du musst ein bisschen wie hier erziehen“ und so dann, sie sagte: „Mein Gott und bei so einem Wetter, deine Kinder drin sitzen müssen und lernen müssen.'. Ich sagte: 'Eh, ich habe nicht gesagt, dass sie nicht nach draußen gehen können, hab ich nie meine Kinder unter Druck so gesetzt. Aber ich hab gesagt, lediglich nur klargemacht, wir sind nicht um Urlaub machen nach Deutschland gekommen.'."

Neben einer weiteren Betonung der Bildungsorientierung Mustafas spricht diese Stelle von Problemen mit strukturellen und direkten rassistischen Anwürfen, denen sich die Familie ausgesetzt sah. Mustafa spricht im Interview erst spät von Problemen, die sie in Deutschland hatten und er erwähnt erst nach Stunden diskriminierendes Verhalten Deutscher ihnen gegenüber. In dieser Stelle scheinen jedoch die Ansprüche und unausgesprochenen Erwartungen auf, mit denen sich die „einzige ausländische Familie“ in der Nachbarschaft konfrontiert sah. Mustafas Insistieren auf den schulischen Fleiß seiner Töchter, sein hohes Bildungsinteresse muss auch vor dem Hintergrund gesellschaftlicher Erwartungen an und Klischeebilder von ,Ausländerfamilien', von denen sich Mustafa, wie schon gezeigt, deutlich abgrenzt, gesehen werden. Diese Erwartungen und Klischees wirken sich direkt auf die Familie aus, zum Beispiel in der übergriffigen Einmischung der Nachbarin in Erziehungsfragen, und sie drohen den schulischen Erfolg zu behindern, wenn z.B. die Grundschullehrerin seine Tochter als nur 'vielleicht fürs Gymnasium geeignet' einschätzt:

„Als die G. in der Grundschule, also mit der Grundschule fertig wurde, die hatte ganz nette Zeugnis, also kann ich dann dir auch zeigen, weil ich alles aufbewahrt habe, und das Kind hat, glaube ich, keine 3, keine einzige 3 auf ihrem Zeugnis, nur 1 und 2. Dennoch schrieb ihre Lehrerin 'vielleicht fürs Gymnasium geeignet' aufs Zeugnis. Darauf habe ich die sie unterrichtende Kollegin angesprochen, ich habe gesagt, das ist irgendwie so widersprüchlich Zeugnis und Beurteilung. Ich sagte zu ihr: 'Du hast dieses Zeugnis geschrieben, das Kind hat nur Einsen und Zweien, was muss man haben, dass man aufs Gymnasium gehen kann?'.“

Mustafa setzt sich für die Belange seines Kindes ein und bezieht gegen die offensichtlich ungerechte und rassistische Beurteilung Stellung. Die besondere Dramatik dieser Situation, die sich letztlich aus der Migration ergibt, fordert hierbei ein besonders deutliches Eingreifen und ermöglicht Mustafa eine ganz klare Positionierung als aktiver Vater.

Mustafa präsentiert sich in seiner biographischen Erzählung als verantwortungsvoller Familienvater. Diese Selbstpräsentation steht in seiner Erzählung gleichberechtigt neben der beruflichen Identität und der Selbstinszenierung als Experte für in Deutschland lebende Türken. Er vermittelt ein Bild von Vaterschaft, das nicht von der Aufgabe des Ernährers sondern vielmehr 
von der des Erziehers geprägt ist. Im großen Interesse an der Bildung seiner Kinder zeigt sich seine Fürsorge. Denn Bildung stellt für ihn den Schlüssel zu einem selbstbestimmten und emanzipiertem Leben dar. Anders ausgedrückt: Mustafa investiert seine Zeit in die Übernahme von care-Aufgaben weil er damit das kulturelle Kapital der Kinder, aber auch das der Familie aufstocken und damit eine höhere Anerkennung in Deutschland wahrscheinlicher machen kann.

\section{Fazit}

Vaterschaft wird, so können wir am Beispiel Mustafas sehen, von Männern der ersten Generation nicht nur im Sinne einer Versorger- und Ernährerrolle verstanden, ebenso wenig treten sie nur als autoritäre Patriarchen in Erscheinung. Wie auch in der Mehrheitsgesellschaft finden sich unter Männern mit Migrationsbiographie unterschiedlichste Formen gelebter Männlichkeiten und Vaterschaft(en). Diese sind jedoch nicht ausschließlich individuell geprägt und Ergebnis familiärer Aushandlungsprozesse, sondern sie sind beeinflusst von den strukturellen Bedingungen der Einwanderungsgesellschaft Deutschland und von diskursiven Effekten. Im Migrationskontext sind diskursive Zuschreibungen, Stereotype und Ethnisierungen wirkmächtig und beeinflussen ebenso wie (migrations)politische Einschränkungen die Konstruktion von Geschlecht sowie die Aufteilung von Arbeit und die Übernahme von care-Verpflichtungen in der Familie. Eine Migration wirkt sich so auch auf das Er- und Ausleben von Vaterschaft und die Übernahme von care-work aus. Väter mit Migrationshintergrund wollen Zeit mit ihren Kindern verbringen, sie sind v.a. am Bildungsweg ihrer Kinder interessiert (vgl. dazu auch Westphal 2000). Durch die Ausübung von Erziehungsaufgaben wird von ethnisierten Männern aber auch innerhalb der Familie nicht das Modell von Männlichkeit in Frage gestellt. Es ist vielmehr die Alltagspraxis, das alltägliche „,doing papa“ in dem sich Umbruchprozesse und Neukonstruktionen vollziehen. Am Beispiel der biographischen Erzählung Mustafas wollten wir zeigen, wie sich Männlichkeit im Migrationskontext durch die Übernahme von care-Aufgaben neu konstituiert. Neben den neuen Herausforderungen, mit denen Männer im Migrationskontext konfrontiert werden und dem Aushandlungsraum, der sich dadurch in Hinblick auf die Gestaltung von Geschlechterverhältnis und Erziehungsvorstellungen ergibt, verweist gerade das Beispiel Mustafas auch darauf, dass das dominante Türkei-Bild revidierungsbedürftig ist. In der Überwindung des Kulturverständnisses, das von Kulturkonflikt und Modernitätsdifferenz geprägt ist, wollten wir hier eine Perspektive auf Männlichkeiten im Migrationskontext anbieten, in der die 
Machtverhältnisse und dementsprechende Rassismuserfahrungen in der Einwanderungsgesellschaft fokussiert werden und zugleich eine Reproduktion der Marginalisierung überwunden wird, indem Vaterschaft und care-Aufgaben in die Konstruktion von (fremden) Männlichkeiten miteinbezogen wird.

\section{Literatur}

Diefenbach, Heike (2007): Kinder und Jugendliche aus Migrantenfamilien im deutschen Bildungssystem. Wiesbaden: VS.

Erel, Umut (2003): Migrantinnen zwischen Anerkennung und Abqualifikation. In: Steyerl, Hito/Gutierrez Rodriguez, Encarnacion (Hrsg): Spricht die Subalterne Deutsch? Migration und postkoloniale Kritik. Köln: Unrast Verlag, S.108-128.

Farrokhzad, Schahrzad (2006): Über Exotinnen, Unterdrückte und Fundamentalistinnen. Zur Konstruktion der „fremden Frau“ in den Medien. In: Butterwegge, Christoph/Hentges, Gudrun (Hrsg.): Massenmedien, Migration und Integration. Wiesbaden: VS-Verlag 2006, S. 53-86.

Gemende, Marion/Munsch, Chantal/Weber-Unger Rotino, Steffi (Hrsg., 2007): Eva ist emanzipiert, Mehmet ist ein Macho. Zuschreibungen, Ausgrenzung, Lebensbewältigung und Handlungsansätze im Kontext von Migration und Geschlecht. Weinheim: Juventa.

Herwartz-Emden, Leonie (2000): Einwandererfamilien: Geschlechterverhältnisse, Erziehung und Akkulturation. Osnabrück: Rasch Verlag.

Hochschild, Arlie Russel (2000): Global Care Chains and Emotional Surplus Value. In: Hutton, Will/Giddens, Anthony (Hrsg.): On the Edge. Living with global capitalism. London: Jonathan Cape, S. 130-146.

Huth-Hildebrandt, Christine (2001): Das Bild von der Migrantin. Frankfurt/Main: Brandes \& Apsel.

Huxel, Katrin (2008): Ethnizität und Männlichkeitskonstruktionen. In: Baur, Nina/Luedtke, Jens (Hrsg., 2008): Die soziale Konstruktion von Männlichkeit. Hegemoniale und marginalisiete Männlichkeit in Deutschland. Opladen: Barbara Budrich, S. 65-78.

King, Vera/Koller, Hans-Christoph (Hrsg., 2006): Adoleszenz-Migration-Bildung. Bildungsprozesse Jugendlicher und junger Erwachsener mit Migrationshintergrund Wiesbaden: VS.

Lutz, Helma (2007): Vom Weltmarkt in den Privathaushalt. Die neuen Dienstmädchen im Zeitalter der Globalisierung. Opladen \& Farmington Hill: Verlag Barbara Budrich.

Lutz, Helma (2009): gender mobile? Geschlecht und Migration in transnationalen Räumen. In: dies. (Hrsg.): gender mobile? Geschlecht und Migration in transnationalen Räumen. Münster: Westfälisches Dampfboot, S. 8-27. 
Parella, Sónia (2008): An Approach to the Transnational practices of Latina Migrants in Spain and its Impact on Transnational Homes. Vortrag gehalten auf dem 1st ISA Forum of Sociology, Barcelona, 5.-8. September 2008, S. 1-25.

Scholz, Sylka (2004): Männlichkeit erzählen. Lebensgeschichtliche Identitätskonstruktionen ostdeutscher Männer. Münster: Westfälisches Dampfboot.

Solé, Carlota/Parella, Sónia/Cavalcanti, Leonardo (2007): Los vínculos económicos y familiares transnacionales. Los migrantes ecuatorianos y peruanos en España. Madrid.

Spies, Tina (i.E.): Subjektpositionen im Diskurs. Eine intersektionelle Analyse von Biographien im Kontext von Migration, Männlichkeit und Kriminalität. Unveröff. Dissertation, Universität Kassel.

Terkessidis, Mark/Yasemin Karakaşoğlu (2006): Gerechtigkeit für die Muslime. In: DIE ZEIT 6, Februar 2006, S.49.

Tunc, Michael (2008): „Viele türlische Männer fliehen von zu Hause“. Mehrfache ethnische Zugehörigkeiten und Vaterschaft im Spannungsfeld von hegemonialer und progressiver Männlichkeit. In: Potts, Lydia/Kühnemund, Jan (Hrsg.): Mann wird man. Geschlechtliche Identitäten im Spannungsfeld von Migration und Islam. Bielefeld: transcript. S. 105-132.

Tunc, Michael (2007): Väter mit Migrationshintergrund zwischen Skandalisierung und Vernachlässigung. Umrisse einer Väterarbeit in der Migrationsgesellschaft. In: Migration und Soziale Arbeit, Jg. 29, Heft 1, S. 33-40.

Tunc, Michael (2006): Vaterschaft in der Migrationsgesellschaft im Wandel. In: Promotionskolleg 'Kinder und Kindheiten im Spannungsfeld gesellschaftlicher Modernisierung' (Hrsg.): Kinderwelten und institutionelle Arrangements. Modernisierung von Kindheit. Wiesbaden: VS.

Volz, Rainer/Zulehner, Paul (2009): Männer in Bewegung. Zehn Jahre Männerentwicklung in Deutschland. Ein Forschungsprojekt der Gemeinschaft der Katholischen Männer Deutschlands und der Männerarbeit der Evangelischen Kirche in Deutschland. Baden-Baden: Nomos Verlagsgesellschaft $<$ http://www.nomos.de $>$.

Weber, Martina (2005): Apartheid im Schulhaus? Konstruktion ethnischer und geschlechtlicher Grenzen im Schulalltag. In: Spies, Anke/Stecklina, Gerd (Hrsg.): Die Ganztagsschule. Herausforderungen an Schule und Jugendhilfe. Band 1: Dimensionen und Reichweiten des Entwicklungsbedarfs. Bad Heilbrunn: Klinkhard, S. 149-171.

Weiß, Anja (2001): Rassismus wider Willen. Ein anderer Blick auf eine Struktur sozialer Ungleichheit. Opladen: Leske und Budrich.

Westphal, Manuela (2000): Vaterschaft und Erziehung. In: Herwartz-Emden, Leonie (Hrsg.): Einwandererfamilien. Geschlechterverhältnisse, Erziehung und Akkulturation. Osnabrück: Rasch, S. 121-204.

Yildiz, Erol (2006): Stigmatisierende Mediendiskurse in der kosmopolitanen Einwanderungsgesellschaft. In: Butterwege, Christoph/Hentges, Gudrun (Hrsg.): Massenmedien, Migration und Integration. Wiesbaden: VS, S. 37-53.

Zulehner, Paul/ Volz, Rainer (1998): Männer im Aufbruch - Wie Deutschlands Männer sich selbst und wie ihre Frauen sie sehen. Ostfildern: Schwabenverlag 